\title{
Molecular beacon-based real-time PCR method for detection of porcine DNA in gelatin and gelatin capsules
}

\begin{abstract}
Background: The pharmaceutical industry has boosted gelatin consumption worldwide. This is supported by the availability of cost-effective gelatin production from porcine by-products. However, cross-contamination of gelatin materials, where porcine gelatin was unintentionally included in the other animal sources of gelatin, has caused significant concerns about halal authenticity. The real-time polymerase chain reaction (PCR) has enabled a highly specific and sensitive animal species detection method in various food products. Hence, such a technique was employed in the present study to detect and quantify porcine DNA in gelatin using a molecular beacon probe, with differences in performance between mitochondrial (cytochrome b gene) and chromosomal DNA-(MPRE42 repetitive element) based porcine-specific PCR assays being compared. Results: A higher sensitivity was observed in chromosomal DNA (MPRE-PCR assay), where this assay allows the detection of gelatin DNA at amounts as as low as $1 \mathrm{pg}$, whereas mitochondrial DNA (CBH-PCR assay) can only detect at levels down to 10 pg of gelatin DNA. When an analysis with commercial gelatin and gelatin capsule samples was conducted, the same result was observed, with a significantly more sensitive detection being provided by the repetitive element of chromosomal DNA. Conclusion: The present study has established highly sensitive DNA-based porcine detection systems derived from chromosomal DNA that are feasible for highly processed products such as gelatin and gelatin capsules containing a minute amount of DNA. This sensitive detection method can also be implemented to assist the halal authentication process of various food products available on the market.
\end{abstract}

Keyword: Gelatin adulteration; Molecular beacon; Porcine DNA; Real-time PCR 